\title{
Simulating Meaning Negotiation Using Observational Language Games ${ }^{\star}$
}

\author{
Tiina Lindh-Knuutila, Timo Honkela, and Krista Lagus \\ Adaptive Informatics Research Centre \\ Helsinki University of Technology \\ P.O. Box 5400, FIN-02015 TKK, Finland \\ firstname. lastnameatkk. fi
}

\begin{abstract}
In this article, we study the emergence of associations between words and concepts using the self-organizing map. In particular, we explore the meaning negotiations among communicating agents. The self-organizing map is used as a model of an agent's conceptual memory. The concepts are not explicitly given but they are learned by the agent in an unsupervised manner. Concepts are viewed as areas formed in a self-organizing map based on unsupervised learning. The language acquisition process is modeled in a population of simulated agents by using a series of language games, specifically observational games. The results of the simulation experiments verify that the agents learn to communicate successfully and a shared lexicon emerges.
\end{abstract}

\section{Introduction}

The acquisition of concepts can be viewed as the process of grounding them in language use. How do concepts form, to begin with, when no language exists in the environment? The relationship between cognitive and linguistic development can be pinpointed to the question: how do conceptual emergence and the formation of names for the concepts connect? An associated important question is how an agreement on the use of words is reached in a community of agents. This process of converging towards a shared use of words is called meaning negotiation.

We study the emergence of associations between concepts and words with help of a computer simulation and consider the hypothesis that concepts are modeled as areas in some conceptual space (see [1]). Furthermore, we utilize the self-organizing map [23] as a model for an agent's conceptual memory [4]. The feasibility of this conceptual memory model is then studied using multi-agent language games, in which agents learn to associate words to meanings in a communicative setting. Prior to learning wordmeaning-associations, a conceptual representation is also learned individually by each agent based on sensory data.

\subsection{Concept Formation}

To be able to model conceptual representations in a cognitive framework, we are using the conceptual spaces theory [1]. According to the theory, concepts can be modeled

\footnotetext{
* This work was supported by the Academy of Finland through Adaptive Informatics Research Centre that is a part of the Finnish Centre of Excellence Programme.
} 
as geometrical areas in a multidimensional conceptual space rather than as symbols or connections among neurons. Gärdenfors proposes that certain neural network or statistical methods, e.g. multi-dimensional scaling or self-organizing maps can be used as a basis for a domain in a conceptual space [1]. It is assumed that concepts are not innate but learned in interaction with the world (consider e.g. [5]).

The self-organizing map (SOM) [3] is a neural network model developed originally in the early 1980s [2]. The SOM model is widely known and the details are not presented here. More information can be found in [3].

We use the self-organizing map as an implementation of a conceptual memory. A domain of a conceptual space of an agent is represented by a self-organizing map trained with observation data. Color data, the RGB values of color pictures, are used for training of the map. Following Gärdenfors' vocabulary, there are then three quality dimensions in this domain of the conceptual space: R(ed), G(reen) and B(lue). The individual selforganizing maps are trained with the color data prior to the language acquisition. After the initial training of the SOM, the map is not changed. This corresponds to a situation in which a child initializes its feature representations based on natural visual data. When an object (color vector) is perceived during the simulation, it is mapped to the trained SOM by finding a unit whose distance to the perceived input is the smallest. This map node is the best-matching unit (BMU) [3]. In our experiments, a cognitive agent does not have any initial ordering or access to the inputs and thus the initialization of the self-organizing map is random. The objects that the agents see belong to eight different categories: What the agents perceive are slightly different instances of these categories. This differs from previous approaches, e.g. [6], where the meanings are presented simply as integers.

The meaning of a word is taken to be a node or a group of (neighboring) nodes in a self-organizing map. Thus, the word is not directly associated with 'something in the world', the referent, which in our case is the perceived data vector but to a representation: The representation of the data vector is the best-matching unit (BMU) in the map.

The association between a word and a concept is implemented by assigning a word to a certain node in a conceptual map. The mapping between words and conceptual map nodes is many-to-many. A node may have several words associated with it and a word may be associated with several nodes. We make a hypothesis that a general agreement among the agents on the word use emerges during the simulation.

\subsection{Language Games}

To model the language acquisition process, we are using simulated language games based originally on the notion of Wittgenstein [7]: Every occasion of language use is a language game. In a language game there is a dialogue between two agents, a speaker and a hearer, within a particular contextual setting. It offers a possibility to study the cultural evolution process of language in subsequent language games instead of subsequent agent generations.

There are several types of simulated language games that have been tested within this framework. Here, we briefly present three of them, the observational game, the guessing game and the selfish game. So far, we have implemented only the observational game in 
which both agents know in advance the topic of the game. The learning is associative: The hearer agent learns the name the speaker uses for that topic.

In the guessing game, both agents are presented a small number of objects. The hearer must then guess which object is the one the speaker refers to with the word it uttered. In the end of the game, the speaker gives some corrective feedback to the hearer telling whether the guess was right or not. In the selfish game introduced by Vogt [8] and Smith [9], the agents do not receive any feedback of the success of their communication. Thus, the learner must infer the meanings of words from their cooccurrences in different contexts or situations. The game is called 'selfish' as in some way the speaker does not care whether the message was correctly understood.

\subsection{Related Work}

In the domain of concept acquisition modeling, Schyns [10] demonstrated how simple concepts could be learned with a modular neural network model. The model has two modules, one for categorizing the input in an unsupervised manner and another module for learning the names in a supervised mode.

Cangelosi and Parisi [11] as well as Grim et al. [12] use feedforward multilayer neural networks whereas we use self-organizing maps [2]3]. Oudeyer [13] models the self-organization of combinatoriality and phonotactics in vocalization systems with a neural network model that is close to the self-organizing map, without any dimensionality reduction. The self-organizing map is based on an unsupervised learning principle which makes it possible to learn efficiently statistical characteristics of input even when no interpretation or classification for the input is given. In a related work [14], the selforganizing maps are used to model the semantic representations of action verb meanings and their clustering, depending on the body part they are related to. Raitio et al. [15] consider the similarity of representations that emerge in unsupervised, self-organization process of neural lattices when exposed to color spectrum stimuli. Self-organizing maps are trained with color spectrum input, using various vectorial encodings for representation of the input. Furthermore, Raitio et al. use the SOM for a heteroassociative mapping to associate color spectrum with color names.

The language game models discussed here were introduced by Steels [16] to study how a coherent lexicon may emerge by means of cultural interactions, individual adaptation and self-organization. The games have been simulated, e.g., in [9], [16], [17] and [18], or implemented in a population of physical robots, e.g., in [8] and [19].

Our work on the multi-agent learning borrows much from the research on the observational games by Vogt [6/178]. Our specific contribution is a detailed model for how perception-to-concept mapping can take place. In [6], there is no categorization at all. In [17], there is a spatial conceptual space which would allow variation around the prototype, but only prototypical colors are used in the simulated world. In our work, we are using the self-organizing map as a spatial conceptual representation, and the colors the agents can perceive contain variation around the prototypes. This means that the mapping between perceptions and concepts is many-to-one: the agents are performing categorization as well.

Learning concepts and language simultaneously is considered in [17]. In our work the conceptual information is learned prior to learning the word-meaning-associations. 
This choice does not reflect a position according which language does not influence the formation of conceptual structures. In these experiments, our aim was to consider a situation in which there is originally little or no categorical information available. Simultaneous and continuous learning of conceptual structures and word-meaning-associations would be possible, for instance, by using the self-refreshing self-organizing map [20].

In [17], the compositionality of language is mainly studied using the iterated learning model (ILM) [21] that contains subsequent generations of adult and child agents. The adults are mainly the teachers and the children are the learners of the emerging language. Neither the ILM or the compositionality of language is considered here.

\section{Methodology}

We implemented the observational game model to study our hypotheses of conceptual modeling and grounding concepts in the language use. In an observational game both agents know in advance the topic of the game. In Vogt's and Steels' robotic experiments this was accomplished by pointing, and later in simulations by using other extra-linguistic information. Our solution is that the agents are able to perceive only one object at the time and this is the topic of the language game. These objects and their properties used in our simulations are presented in more detail later.

Each agent has a conceptual memory based on a SOM and a lexicon. The lexicon contains all words that are in the agent's vocabulary, and information on which nodes of the SOM they are associated to. It also contains a counter value for the word-node pair describing how successfully a word has been used to express a meaning previously. The minimum value of the counter is zero and the maximum value we have used is twenty.

\subsection{Algorithm of the Observational Game}

Each language game in the simulation proceeds in the following way.

1. Two agents are chosen randomly from the population of agents. One is arbitrarily assigned the role of the speaker, the other the hearer.

2. The topic of the language game is chosen randomly from the set of topics and shown to both agents.

3. Both the speaker and the hearer search for a node in their own conceptual map that best matches the topic (the BMU).

4. The speaker searches for the word that could match the topic. The search is performed in a neighborhood of the best-matching unit (BMU) defined by $R$, which is an integer, $R \geq 1$. The process of the word search is described later in more detail. If no possible word is found, a new word is invented and associated to the BMU. This word is communicated to the hearer.

5. The hearer searches for a set of possible words that could denote the topic. The search is performed in a similar way as in the case of speaker, but instead of one best word, all the words that are found are returned. If the word the speaker has uttered belongs to this set, the language game is considered a success, otherwise the game fails. 
6. In case of a successful game, both the speaker and the hearer increase their counter for the word by one. If the uttered word was not among the labels of the BMU, it is then added to it. The maximum value of the counter is set to 20 .

7. If the game fails, the speaker decreases by one the counter of the uttered word. The minimum value allowed for the counter is zero. If the speaker's BMU node did not contain any label but the word was instead found from the neighborhood, the word is not added to the BMU node of the speaker. The hearer labels its BMU with the spoken utterance in any case.

\subsection{Utterances}

In the simulation framework, each word is a discrete symbol. A word is a string of characters generated from a simple artificial language and it is uttered when needed. If there does not exist a word that could be used to denote the topic of the conversation, a novel word is generated from the language.

In our experiments, a limited artificial language is used. In this language, there are words that contain either four or six characters. The alphabet contains vowels $V=$ $(a, e, i)$ and consonants $C=(b, c, d, f, g, h)$. In total, the alphabet consists of nine letters. Each word of this language begins with a consonant which is then followed by a vowel. The pattern is repeated either once or twice, so all the words are either of the form 'CVCV' or 'CVCVCV'.

In many previous simulations, e.g., [11], the set of words that could be used was small and fixed. In these simulations the set of words is finite but open: new words can enter to the simulation, whereas the number of topics of the language games is fixed.

\subsection{Word Search Process Using the SOM}

When an agent is shown the topic, i.e. the vector containing the features of the topic, it finds a prototype vector from its conceptual memory that best matches the given input vector. This prototype vector is called the best-matching unit (BMU). The prototype vectors can be assigned labels, the words. The search algorithm searches for words used to label the BMU and nearby nodes that are within the radius $R$ in the neighborhood of the BMU. This means that if there already exists a word associated to a similar enough concept (i.e. there is a word in the neighborhood $\mathrm{R}$ of the node), this word can be used to name the new object even if the BMU itself was not associated with any word. As the neighborhood is considered, later in the simulation there are often several possible words the speaker could use. The uttered word is the one that had been used most successfully earlier in the neighborhood of the BMU, defined by the counter values associated to each word-node pair. In case of multiple words with the same count, one of them is selected randomly. If the set of words is empty, a new word is generated and uttered.

The hearer searches for the possible words in a similar way to the point of finding a set of words. The only difference is that there is no need to select the best word but the set of possible words is compared to the word uttered by the speaker. In general, the competing word-meaning pairs are considered to be either synonymous or polysemous, both being features of natural languages as well. Here synonymy is considered as a relative term: words or phrases may have similar meaning to some degree. 


\section{Experiments}

The purpose of our experiments was two-fold: The first goal of the experiments was to verify the hypothesis that the agents are able to develop an emergent and shared lexicon by engaging in the language games, while using the conceptual memory model based on the self-organizing map. Secondly, we were studying the association between the map nodes and the utterances created by agents and how the areas that are named with the same word are formed. To test how the varying parameters affect the overall learning results, experiments were conducted with different population sizes and varying the search radius.

In all experiments, ten simulations were run with different random seeds for 5000 language games. There were three measures used to evaluate the outcome of the simulations. The communication success was calculated after every language game. The coherence and specificity measures were calculated after every 250 games, and the size of the lexicon was calculated in the end of the simulation. The coherence measure indicates whether a certain word is utilized coherently among the agents to denote a certain meaning in the community. Specificity is a measure that decreases if two meanings are referred to with the same word. These measures are explained later in more detail.

\subsection{Color Data and Language Game Topics}

The agents' conceptual maps were trained with three-dimensional color data vectors. Components of the vector were $\mathrm{R}(\mathrm{ed}), \mathrm{G}($ reen) and $\mathrm{B}(\mathrm{lue})$ values of a pixel in a color picture. The color data consisted of ten pictures - one for each agent. The size of these pictures was $100 \times 100$ pixels. Thus, for each agent, the size of the training set was 10000 samples, the total number of pixels in the training picture.

The color pictures were created by drawing filled ellipses and rectangles in color onto a white background. As a starting point, we were using the RGB values of eight different 'prototypical' colors: black, blue, green, cyan, red, magenta, yellow and white. To get less spiky distributions for each color, uniformly distributed noise was added independently to each of the three color channels (RGB) of the picture. The level of noise was set to $20 \%$ of the total color range.

In the experiments, a hexagonal map topology was used. The size of the used map was $16 \times 12$ map nodes. The maps were initialized randomly. All the maps were trained in a batch training mode. [3]

A set of 400 additional color pictures were utilized as language game topics. They were generated in similar manner as the training data, but were not part of the training set. For the purpose of limiting computational workload, the size of a picture used as a topic was limited to $20 \times 20$ pixels. The topic was chosen randomly from this group for each game.

\subsection{Evaluation Measures}

To evaluate the agent learning, we utilized four measures: communication success, coherence, specificity and lexicon size.

In a successful language game, the word the speaker used to denote a given topic was found among the words that also the hearer associated with the topic. Communication 
success is a longer-term outcome of the language games. It is defined in a similar way as in [6] 18] as the average number of correctly played games in the past 100 games or less if no 100 games had been played yet. It is calculated after every language game.

Coherence is a population measure, which measures whether a certain word is utilized coherently among the agents to denote a certain meaning in the community. It is the rate in which agents would produce a certain word to express a particular meaning. The coherence measure calculation used in this work is taken from [18]. For each topic, a fraction of agents that has the same word as a preferred word is calculated and the maximum fraction is taken. This is then averaged over all topics. If an agent does not have a word to express a certain meaning, the coherence is set to zero.

Specificity measure developed by De Jong was used: "Specificity indicates to what degree the words an agent uses determine the referent that is the subject of communication" [18]. Specificity decreases if two meanings are referred to with the same word. Thus it also describes the degree of polysemy in the lexicon: the higher the specificity, the less polysemy there is. Here, the specificity based on preferred words [18] is used.

For each agent, $A_{i}$, the specificity, $\operatorname{spec}\left(A_{i}\right)$, is calculated using the following formula:

$$
\operatorname{spec}\left(A_{i}\right)=\frac{n_{s}^{2}-\sum_{k=1}^{n_{s}} f_{k}}{n_{s}^{2}-n_{s}},
$$

where $n_{s}$ is the number of referents, and $f_{k}$ is frequency of the word related to the concept that describes how many referents the word is associated to. The specificity of the population, spec, is then defined as the average specificity of the agents:

$$
\text { spec }=\frac{\sum_{i=1}^{n_{a}} \operatorname{spec}\left(A_{i}\right)}{n_{a}},
$$

where $\operatorname{spec}\left(A_{i}\right)$ is the specificity of an agent and $n_{a}$ is the number of agents. Additionally, if there is no word to denote a certain referent (or topic), it means that the referent cannot be separated from other referents.

In the end of each simulation run, the average size of lexicon was also calculated. In the lexicons, there were also words having a zero counter value, which means that they were not used successfully at all in the course of the simulation. This means that an agent had come up with a new word but this word had not been used successfully and another one had been preferred since. To better show the difference in lexicon sizes, the average size of the lexicon was calculated both before and after the removal of the non-used words. The average lexicon size was calculated as a mean of individual agent lexicon sizes.

\section{Results}

In all our experiments, the results were averaged over 10 simulation runs. All simulations were run for the population sizes of 2, 4, 6, 8 and 10 agents. The results with varying population size are presented in Fig. 1 1 The communication success (Fig. 1 a) climbs quickly close to the maximum value of 1.0. The communication success level 1.0 indicates that each of the previous 100 language games ended successfully. The 

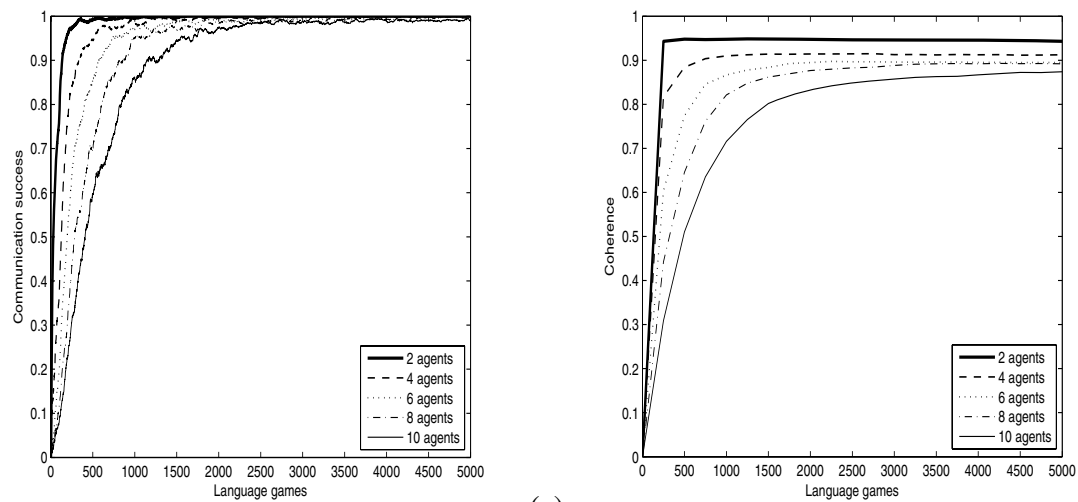

(a)
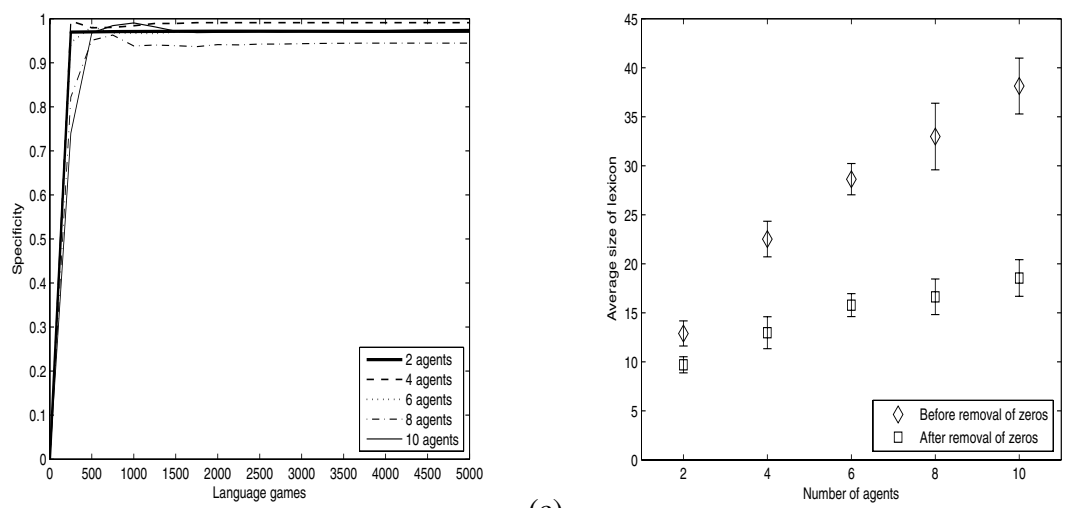

(c)

Fig. 1. Communication success (a), coherence (b), specificity (c) and the lexicon size (d) for varying population size, when $R=2$ and the map size is $16 \times 12$

larger the population is, the longer it takes to reach the maximum level, but even in the case of 10 agents the level of 0.9 is reached after approximately 1250 games.

The coherence level (Fig. 1 b) increases also quite rapidly. In the case of population size of 10 agents, the coherence level reaches 0.86 , whereas with smaller population sizes it is 0.9 or higher. Thus, as simulation advances, the agents begin to use more and more the same word to denote the same referent, thus forming a shared vocabulary. As pointed out earlier, the coherence does not say anything whether the agents are using the same word to denote each referent. The specificity (Fig. 1 c), rises over 0.9 already after 250 games with each population size. Thus, there seems to be little polysemy in this case: The agents are using a separate word for each prototypical color.

The average size of the lexicon (Fig. 1 d) stays between 10 and 17, rising only a little as a function of the population size. The whiskers describe the amount of standard deviation in the average values. The size of the lexicon, all words included, is a function of the population size.

The results seem promising: They clearly show that the agents can develop a shared lexicon to denote the objects they perceive. The size of the population seems only to 
affect on how quickly the communication success and coherence levels grow. The reason behind this is that in larger populations, it takes longer for the whole population to develop a common vocabulary, as in each language game, there are always only two agents playing. As such, though, the relationship between population size and learning speed is known well through a number of earlier studies.

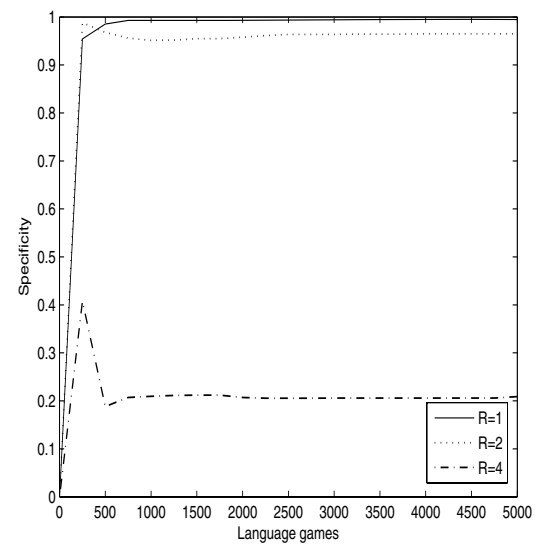

Fig. 2. The specificity values when the search radius was changed

To study how the size of the search radius, $R$ affects the results we repeated the experiment using varying search radii. The communication success and coherence behaved similarly to the previous experiment. Communication success and coherence values increase rapidly as more language games are played. The specificity measure reveals something interesting (see Fig. 2 presenting results of using six agents). With the smaller radii, the specificity rises again over 0.95 after only 500 language games. But when using the largest radius, $R=4$, the specificity first rises to the level of 0.4 and then drops to the level of 0.3 as the simulation advances: This indicates that in the beginning of the simulation there is some variation in the names for the language game topics. As the simulation advances only few names are gaining popularity: In the end the agents use only a couple of names to denote all the topics.

Examples of conceptual maps from simulations with six agents are shown in Fig. 3 They show the conceptual space of one agent after the simulation run. The selforganizing map is labeled with the words that have been used during the simulation. The figure on the left shows a case where $R=1$ and in the figure on the right, $R=4$. A thorough visual interpretation of these figures may be hard since the language is made up by the agents themselves and in the each simulation run, the self-organizing maps look different due to random initialization. Thus, they are shown for general interest only. In the map on the right, there is one dominant word, 'bihi' that is used to label almost everything. In the figure on the left-hand side, there are different words that seem to be dominant in different areas. By only inspecting the visualization of the map, it is impossible to say which words are used the most.

The low specificity value does not affect the communication success at all. The agents just have one or two common words to denote everything. The large search 

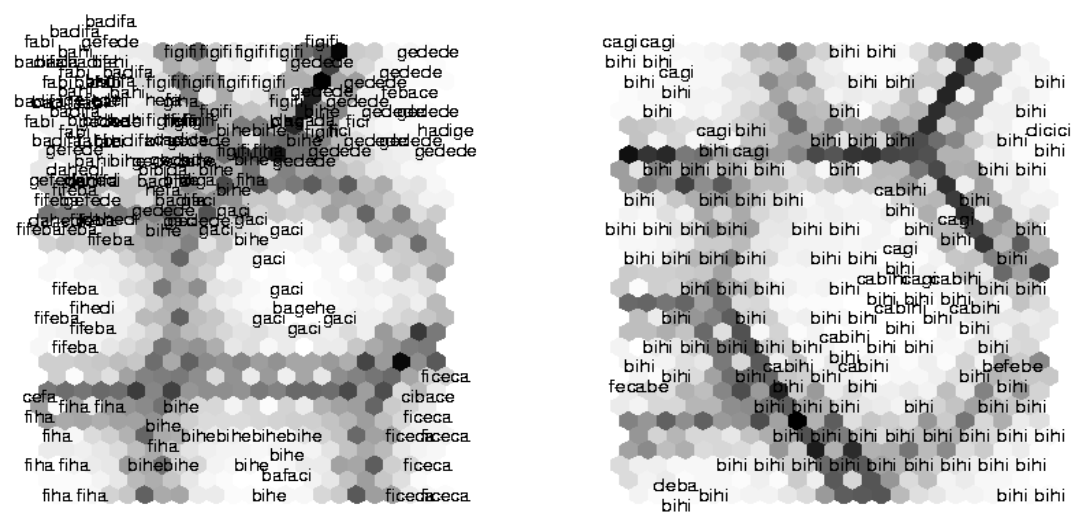

Fig. 3. Two example conceptual maps from simulations with six agents. On the left, a small search radius, $R=1$, was used. On the right, $R=4$. The shades of gray denote distances in the original space: The larger the distance, the darker the color. One can see that there are eight clusters on each map which corresponds to the number of prototypical colors used in the input.

radius makes it possible to use a word already in the lexicon for observed topics, even if the best-matching unit related to that word is very far from the new observation. This is caused by the nature of the observational game: There is no need to make distinctions between observations and thus nothing is inhibiting this behavior, as communication success stays high, which was also noticed by Vogt (e.g., [6]).

\section{Conclusions and Discussion}

In this article, some aspects of language acquisition and conceptual modeling have been considered. In the field of conceptual modeling, the conceptual spaces theory by Gärdenfors [1] has been adopted. The theory provides a medium between the symbolic level of words and the sensory level of 'raw' sensations. The notion of distance provides a possibility to make graded conceptual system: The more prototypical instances of a concept can be seen as more central than the less prototypical instances of the category.

We have described how simulated agents map the perceptions to their conceptual map and associate utterances to these maps. The topological ordering of the maps gives rise, for instance, to a rather natural way for finding names for previously unnamed topics. As a model for shared vocabulary acquisition, different types of language games were discussed in this article. A computer simulation to model one of them, the observational game, was implemented based on the work presented in [6], [8] and [16].

The results of the experiments show clearly that when using the observational game model and the SOM-based conceptual maps (1) the agents learned to communicate successfully on the topics of the games, and (2) a shared lexicon was developed during the simulations. According our definition of successful communication, the agents are also able to communicate successfully and develop a shared lexicon based on adaptation. 
Implementing the guessing game and the selfish game described in Section 1.2 in addition to the observational game, would be useful. The language is somehow redundant in the observational game framework: Both the speaker and the hearer know for sure what the topic of the game is and there is no need to have distinct words to separate different objects. One could study if the need to be able to identify the topic from a group of objects is a pressure enough to prevent the agents of calling all referents with the same word as demonstrated in our experiment using a large search radius. Even though comparisons between the different games have been conducted earlier (e.g., [6]17]), we find it important to determine how the use of the SOM-based conceptual memory influences the results in the case of the other two language games.

In future research, the use multiple maps can be be studied, each for a different conceptual domain (consider, for instance, [14]). In that kind of model, a more complex concept say 'apple', would have properties in different domains, for instance, 'green' (or 'yellow', or 'red') in the color domain, 'round(ish)' in the shape domain, and 'sweet' in the taste domain. Gärdenfors [1] argues that the properties that are relevant in the case of a particular concept depend on the context. The context-dependency would then cause some properties to be more salient in that context. It is possible that these saliencies could be modeled with some kinds of weights. Possibly, the research could be expanded further to somewhat complex concepts: to those with properties extending to different domains of conceptual spaces.

\section{References}

1. Gärdenfors, P.: Conceptual spaces: The Geometry of Thought. MIT Press (2000)

2. Kohonen, T.: Self-organized formation of topologically correct feature maps. Biological Cybernetics 43 (1982) 59-69

3. Kohonen, T.: Self-Organizing Maps. Springer Series in Information Sciences. Springer (2001)

4. Honkela, T.: Neural nets that discuss: a general model of communication based on selforganizing maps. In Gielen, S., Kappen, B., eds.: Proceedings of ICANN'93, International Conference on Artificial Neural Networks, Amsterdam, the Netherlands, Springer-Verlag, London (1993) 408-411

5. Steels, L.: Perceptually grounded meaning creation. In Tokoro, M., ed.: ICMAS96, AAAI Press (1996)

6. Vogt, P., Coumans, H.: Investigating social interaction strategies for bootstrapping lexicon development. Journal of Artificial Societies and Social Simulation 6(1) (2003)

7. Wittgenstein, L.: Philosophical Investigations. The Macmillan Company (1963)

8. Vogt, P.: Lexicon Grounding in Mobile Robots. PhD thesis, Vrije Universiteit Brussel (2000)

9. Smith, A.D.M.: Establishing communication systems without explicit meaning transmission. In Kelemen, J., Sosik, P., eds.: Proceedings of the 6th European Conference on Artificial Life, ECAL 2001, Berlin, Heidelberg, Springer-Verlag (2001)

10. Schyns, P.: A modular neural network model of concept acquisition. Cognitive Science 15 (1991) 461-508

11. Cangelosi, A., Parisi, D.: The emergence of a 'language' in a population of evolving neural networks. Connection Science 10(2) (1998) 83-97

12. Grim, P., Kokalis, T., Tafti, A., Kilb, N.: Evolution of communication with a spatialized genetic algorithm. Evolution of Communication 3(2) (1999) 
13. Oudeyer, P.Y.: The self-organization of combinatoriality and phonotactics in vocalization systems. Connection Science 17(3-4) (2005) 325-341

14. Wermter, S., Elshaw, M.: Learning robot actions based on self-organising language memory. Neural Networks 16 (2003) 691-699

15. Raitio, J., Vigário, R., Särelä, J., Honkela, T.: Assessing similarity of emergent representations based on unsupervised learning. In: Proc. of International Joint Conference on Neural Networks (IJCNN 2004), Budapest, Hungary (2004) 597-602

16. Steels, L.: Emergent adaptive lexicons. In Maes, P., ed.: SAB96, Cambridge, MA, MIT Press (1996)

17. Vogt, P.: The emergence of compositional structures in perceptually grounded language games. Artificial Intelligence 167(1-2) (2005) 206-242

18. De Jong, E.: Autonomous Formation of Concepts and Communication. PhD thesis, Vrije Universiteit Brussel (2000)

19. Steels, L., Vogt, P.: Grounding adaptive language games in robotic agents. In Husbands, C., Harvey, I., eds.: Proceedings of the Fourth European conference on Artificial Life, Camridge, MA and London, MIT Press (1997)

20. Pöllä, M., Lindh-Knuutila, T., Honkela, T.: Self-refreshing SOM as a semantic memory model. In: Proceedings of AKRR'05, International and Interdisciplinary Conference on Adaptive Knowledge Representation and Reasoning, Espoo, Finland (2005) 171-174

21. Kirby, S.: Spontaneous evolution of linguistic structure: an iterated learning model of the emergence of regularity and irregularity. IEEE Transactions on Evolutionary Computation 5(2) (2001) 102-110 